\title{
Evaluation of ${ }^{18}$ F-2-deoxy-2-fluoro-glucose positron emission tomography for gastric cancer screening in asymptomatic individuals undergoing endoscopy
}

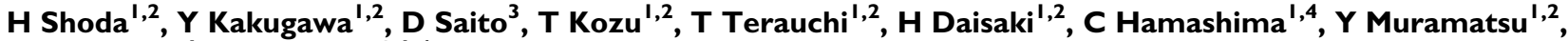 N Moriyama' and $\mathbf{H}$ Saito*, ${ }^{*, 4}$}

'Research Center for Cancer Prevention and Screening, National Cancer Center, Tsukiji 5-I-I, Chuo-ku, Tokyo I04-0045, Japan; ${ }^{2}$ Cancer Screening Division, Tsukiji 5- I - I, Chuo-ku, Tokyo 104-0045, Japan; ${ }^{3}$ Endoscopy Division, National Cancer Center Hospital, Tsukiji 5- I-I, Chuo-ku, Tokyo I04-0045, Japan; ${ }^{4}$ Cancer Screening Technology Division, Tsukiji 5-I-I, Chuo-ku, Tokyo 104-0045, Japan

\begin{abstract}
${ }^{18}$ F-2-deoxy-2-fluoro-glucose Positron Emission Tomography (FDG-PET) has been recently proposed as a promising cancerscreening test. However, the validity of FDG-PET in cancer screening has not been evaluated. We investigated the sensitivity of FDGPET compared with upper gastric endoscopy in gastric cancer screening for asymptomatic individuals. A total of 286I consecutive subjects (1600 men and 126I women) who were asymptomatic and who underwent both FDG-PET and upper gastrointestinal endoscopy between I February 2004 and 31 January 2005 were included in this study. Both endoscopists and a radiologist were unaware of the results of the other diagnostic tests. The FDG-PET images were examined using criteria determined by the pattern of FDG accumulation. Sensitivity and specificity of FDG-PET were calculated compared with endoscopic diagnosis as the gold standard. Among 286 I subjects enrolled in the study, there were 20 subjects with gastric cancer, of whom 18 were TI in depth of cancer invasion. Positive FDG-PET results were obtained only in 2 of the 20 cancer subjects. The calculated sensitivity and specificity for overall gastric cancers were 10.0\% (95\% confidence interval (Cl): 1.2-31.7\%) and 99.2\% (95\% Cl: 98.8-99.5\%), respectively. ${ }^{18} \mathrm{~F}$-2-deoxy-2-fluoro-glucose Positron Emission Tomography was poorly sensitive for detection of gastric cancer in the early stages. British Journal of Cancer (2007) 97, I493-1498. doi:I0.I038/sj.bjc.6604062 www.bjcancer.com
\end{abstract}

Published online 27 November 2007

(c) 2007 Cancer Research UK

Keywords: gastric cancer; screening; endoscopy; FDG-PET; sensitivity

${ }^{18}$ F-2-deoxy-2-fluoro-glucose Positron Emission Tomography (FDG-PET) is a technique that reflects the changes in glucose metabolism in tumour cells, and has been widely used clinically to differentiate between benign and malignant tumours (Rigo et al, 1996), to assess the effectiveness of chemotherapy or radiotherapy (Kelloff et al, 2005), and to predict prognosis (Oshida et al, 1998; Oku et al, 2002). The potential of FDG-PET for early detection of cancer has been investigated because the test enables scanning of the whole body simultaneously and non-invasively. Because of this advantage, there has been considerable enthusiasm for PET screening in Japan (Yasuda and Ide, 2005). About $60 \%$ of facilities in Japan that are equipped with PET offer PET examinations to individuals who hope to undergo cancer screening (Yasuda and Ide, 2005).

Gastric cancer is one of the most important cancers in terms of anticancer strategy because it ranks second in cancer mortality in Japan (World Health Organization Statistics, 2006). There are many other countries with patients at high risk for gastric cancer, such as those in Central and South America, Asia, and Eastern Europe. Although gastric cancer has decreased in most of the

* Correspondence: Dr H Saito, Research Center for Cancer Prevention and Screening, National Cancer Center, Tsukiji 5-I-I, Chuo-ku, Tokyo 104-0045, Japan; E-mail: hrsaito@ncc.go.jp

Received 22 May 2007; revised 4 September 2007; accepted I October 2007; published online 27 November 2007 developed countries, its prevention remains an important issue in those countries. For early detection of gastric cancer, X-ray examination with a barium meal has been employed in Japan (Fukao et al, 1995). Efficacy of this kind of screening program has been strongly suggested, although the studies are observational (Oshima et al, 1986; Fukao et al, 1995; Mizoue et al, 2003). The problem with the program is that the screening test is somewhat invasive in terms of complications such as constipation being frequently seen and mis-swallowing of barium into the trachea (Tamura et al, 1985; Sugahara et al, 1992). On the other hand, with FDG-PET, there is almost no such inconvenience for screenees. For these reasons, FDG-PET has been explored as a potential alternative to the present screening test for gastric cancer in Japan. However, the validity of FDG-PET in cancer screening remains to be evaluated. Although the sensitivity of FDG-PET for gastric cancer is reported to be from 60 to $94 \%$, most subjects evaluated in existing reports were limited to patients with advanced gastric cancers or recurrent cancers (Yeung et al, 1998; De Potter et al, 2002; Stahl et al, 2003; Yoshioka et al, 2003; Mochiki et al, 2004; Chen et al, 2005; Yun et al, 2005). There has been no study to measure screening sensitivity of FDG-PET for gastric cancer in average risk individuals. Therefore, in the present study, we investigated the sensitivity of FDG-PET for gastric cancer in asymptomatic individuals who underwent FDG-PET as well as screening upper gastrointestinal endoscopy, which served as the gold standard in calculating the sensitivity of FDG-PET. 


\section{MATERIALS AND METHODS}

\section{Subjects and study design}

The Research Center for Cancer Prevention and Screening (RCCPS), National Cancer Center (NCC), Tokyo, started the onearm prospective cohort study designed to evaluate the efficacy of multiphasic cancer screening programs in 1 February 2004 (Hamashima et al, 2006). Details of the screening programs are described elsewhere (Hamashima et al, 2006). The screening programs consisted of upper and lower gastrointestinal endoscopy or X-ray examinations and other imaging modalities such as a chest helical CT examination. These examinations were performed during the 2-day course of the screening program. Individuals who were found to have cancer lesions were treated at the National Cancer Center Hospital. Participants were enrolled nationwide. Screenees were asymptomatic men 50 years or over and women 40 years or over who gave signed informed consent approved by the Ethics Committee for Clinical Research of the NCC. Subjects who were diagnosed as having any cancer within the past 1 year, or those who had been treated for cancer or followed-up for precancerous diseases based on self-reporting were excluded. All participants responded to a questionnaire describing many issues concerning life style, family history, and previous examinations within a year (Hamashima et al, 2006). Individuals were to be followed up annually by a questionnaire on health status, diagnostic examinations (including results), and other relevant data.

The study population in the present study was defined as consecutive screenees who underwent both FDG-PET and gastrointestinal endoscopy between 1 February 2004 and 31 January 2005 within the screening program at the RCCPS. There were a total of 2911 individuals who underwent FDG-PET, among whom 2892 individuals, including 1626 men and 1266 women, also had gastric endoscopy and thus met the criteria for inclusion. Thirty-one individuals were excluded who had undergone gastrectomy. After excluding these subjects, the study population of 2861 participants, including 1600 men and 1261 women, was included in the analyses.

The endoscopic findings and images were examined by three skilled endoscopists (HS, YK, and TK) without any knowledge of FDG-PET findings. The FDG-PET images were examined by one expert radiologist specialising in nuclear medicine (TT), who had no information about the endoscopic findings. Findings and diagnoses were recorded separately by endoscopists and the radiologist on the electronic record system at the RCCPS to create the database of the participants. After the records were completed, findings from the two modalities were compared by either of the two investigators (HS and YM) to identify true positives and false negatives from FDG-PET results for gastric cancer based on endoscopic findings as the gold standard. Gastric cancer subjects were defined as those who were diagnosed as having gastric cancer at the time of screening or on additional endoscopy performed within 1 month after the screening.

The study protocol was approved by the Ethics Committee for Clinical Research of the NCC.

Information on cancers other than gastric cancers detected in the background population from which the present study population was drawn was described previously (Hamashima et al, 2006).

\section{${ }^{18}$ F-2-deoxy-2-fluoro-glucose Positron Emission Tomography procedure}

The FDG-PET images were obtained using two multi-ring PET scanners (ECAT Accel, Siemens, Knoxville, TN, USA) with a transaxial resolution of $6.2 \mathrm{~mm}$ at full-width half-maximum. Subjects were required to fast for at least $5 \mathrm{~h}$ before the PET scan.
Sixty minutes after injection of $2.78 \mathrm{MBq} \mathrm{kg}^{-1}$ of FDG that was produced in our radiopharmacy, emission and transmission scans were obtained from the head to the inguinal region. A threedimensional emission scan was acquired in eight or nine bed positions for $2 \mathrm{~min}$ per position, followed by a two-dimensional transmission scan for $1 \mathrm{~min}$ per position to correct for photon attenuation using a $68 \mathrm{Ge} / \mathrm{Ga}$ rod source. Images were reconstructed iteratively (ordered-subset expectation maximisation method, two iterations, eight subsets).

The standardised uptake value (SUV) was semiquantitated in the cases with uptakes suspected of being abnormal. The SUV can be calculated as the ratio of the FDG uptake in a small region of interest (placed over the lesion in an attenuation-corrected image) to the administered activity adjusted for the body weight of the patient (Bombardieri et al, 2003).

\section{Assessment of FDG-PET findings}

Criteria for the assessment of FDG-PET findings for gastric lesions vary among facilities despite the widespread use of the guidelines for the FDG-PET procedure, mainly due to the difficulties caused by physiological uptake in the stomach. Because there are no established criteria for assessing FDG-PET findings, we determined the following criteria based on previous reports (Cook et al, 1996; Gordon et al, 1997; Shreve et al, 1999; Koga et al, 2003): (1) positive pattern 1 - spotty or focal accumulation that was stronger than the uptake in the liver (Figure 1A); positive pattern 2 - any accumulation in the area of the lower stomach (Figure 1B). This category was based on a report by Koga et al (2003), suggesting that physiological gastric FDG uptake is significantly higher at the oral end than the anal end, and that a stronger gastric FDG uptake at the anal end might therefore be suggestive of a pathological uptake. (2) negative pattern 1 - no definite accumulation in the stomach (Figure 1C); negative pattern 2 - diffuse accumulation in the stomach, considered to be a normal physiological uptake (Figure 1D). The judgment of FDG-PET accumulation was made based only on PET without CT scan. Positive whole body FDG-PET findings were obtained in $9 \%$ of 2911 subjects who had FDG-PET examinations. Approximately one-fourth of those with positive FDG-PET required further investigation in addition to the examinations included in the screening program. Detailed information will be described elsewhere.

\section{Upper gastrointestinal endoscopy}

All subjects were administered a $100 \mathrm{ml}$ solution containing $1 \mathrm{~g}$ of Pronase and $1 \mathrm{~g}$ of sodium bicarbonate to remove mucus and bubbles on the gastric mucosa before examination. The antiperistaltic (20 mg of scopolamine butylbromide or $1 \mathrm{mg}$ of glucagon) and sedative $(17.5-35 \mathrm{mg}$ of pethidine hydrochloride or $2-10 \mathrm{mg}$ of midazolam) agents were injected intravenously except when they were contraindicated. We used standard commercial video endoscopic equipment (GIF TYPE H-260 or Q260; Olympus Co., Tokyo, Japan). Endoscopic images were obtained and recorded in a standardised pattern, which covered the entire gastric mucosa in about 50 shots. We added chromoendoscopy with $0.2 \%$ solution of indigo-carmine in all subjects after conventional observation. All lesions that appeared potentially malignant were biopsied for histopathological examination. The location, description of lesions, and diagnosis were recorded just after the gastrointestinal endoscopy. Size of cancer lesions was measured on the surgically or endoscopically resected specimen. Endoscopic images were reviewed primarily on the same day by three endoscopists (HS, $\mathrm{YK}$, and $\mathrm{TK}$ ) to determine whether there were any lesions overlooked during endoscopy. If any suspicious findings were suggested to have been overlooked, the screenees were recommended to undergo an additional endoscopy. 


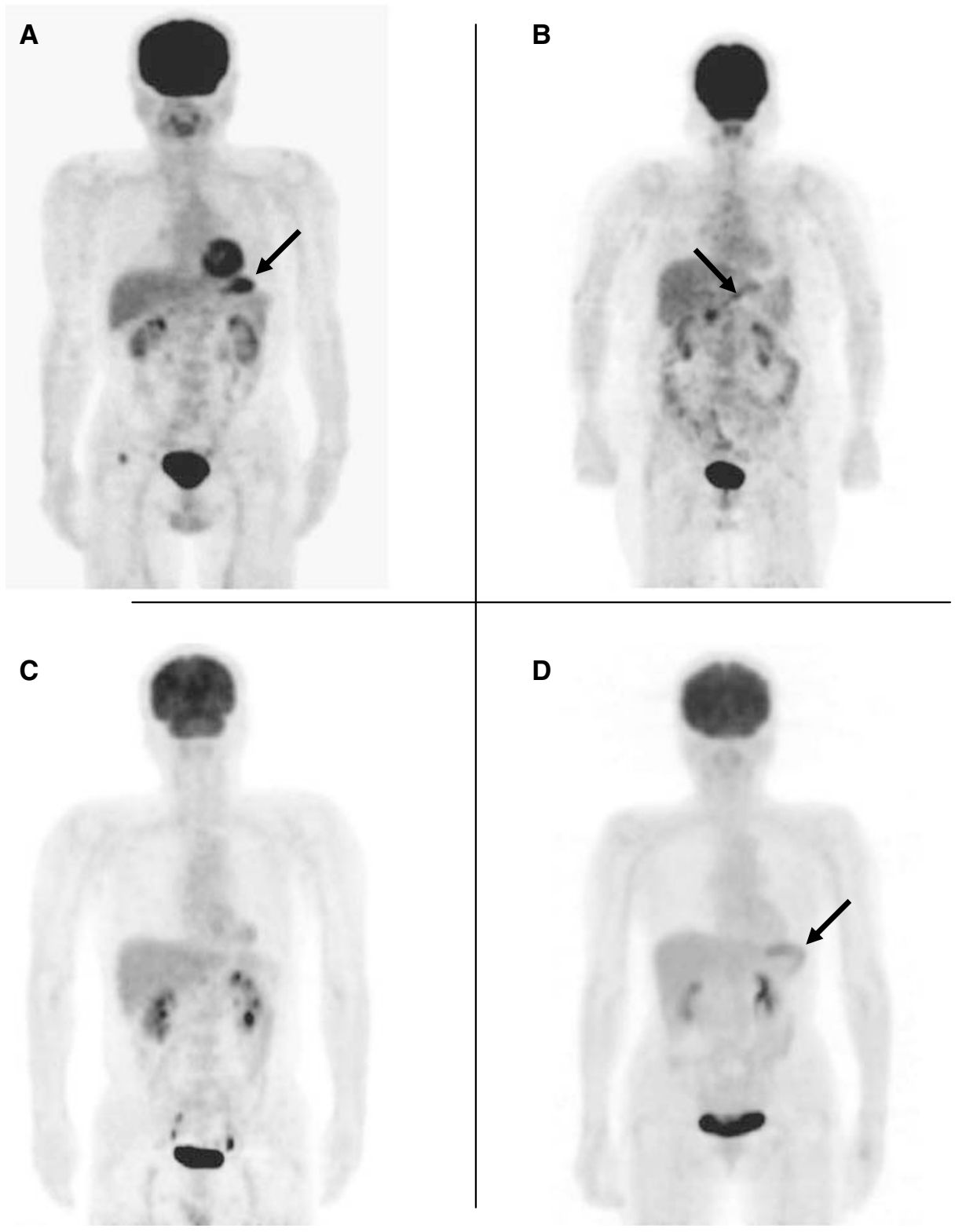

Figure I Assessment of FDG-PET findings. (A) PET scan demonstrates spotty or focal accumulation that is stronger than the uptake in the liver (arrow). (B) PET scan demonstrates focal accumulation in the area of the lower stomach (arrow). (C) PET scan demonstrates no definite accumulation of FDG in the stomach. (D) PET scan demonstrates diffuse accumulation (normal physiological accumulation) of FDG in the stomach (arrow).

\section{Histopathological findings}

The final pathological diagnosis was confirmed from specimens resected surgically or endoscopically. The depth of cancer invasion was recorded according to the TNM clinical classification (Sobin and Wittekind, 1997). Two pathologists interpreted the histopathologic features and when there was a disagreement, a senior pathologist reviewed the features to resolve the disagreement.

\section{Statistical analyses}

The Student's $t$-test was used to assess the difference in the mean age between gastric cancer subjects and those without gastric cancer or between male and female subjects. Statistical significance for comparison of items other than age between subjects with gastric cancer and subjects without gastric cancer was assessed by $\chi^{2}$ test. The difference in SUV between true positives and false positives was also analysed by the Student's $t$-test. $P$-values $<0.05$ were considered statistically significant and $95 \%$ confidence intervals (CIs) were calculated based on a binominal distribution.

\section{RESULTS}

The characteristics of the subjects enrolled in the study are shown in Table 1. Among 2861 subjects enrolled in the study, gastric cancers were detected by gastrointestinal endoscopy in 20 subjects, including 18 men and 2 women. The mean age of all subjects was 59.8 years old, and there was no statistically significant difference between subjects with gastric cancer and subjects without gastric cancer. Males were older than females both among subjects with gastric cancer and subjects without gastric cancer. The proportion of males to females was significantly higher for subjects with gastric cancer than for subjects without gastric cancer (Table 1). 
Table I Characteristics of subjects enrolled in this study

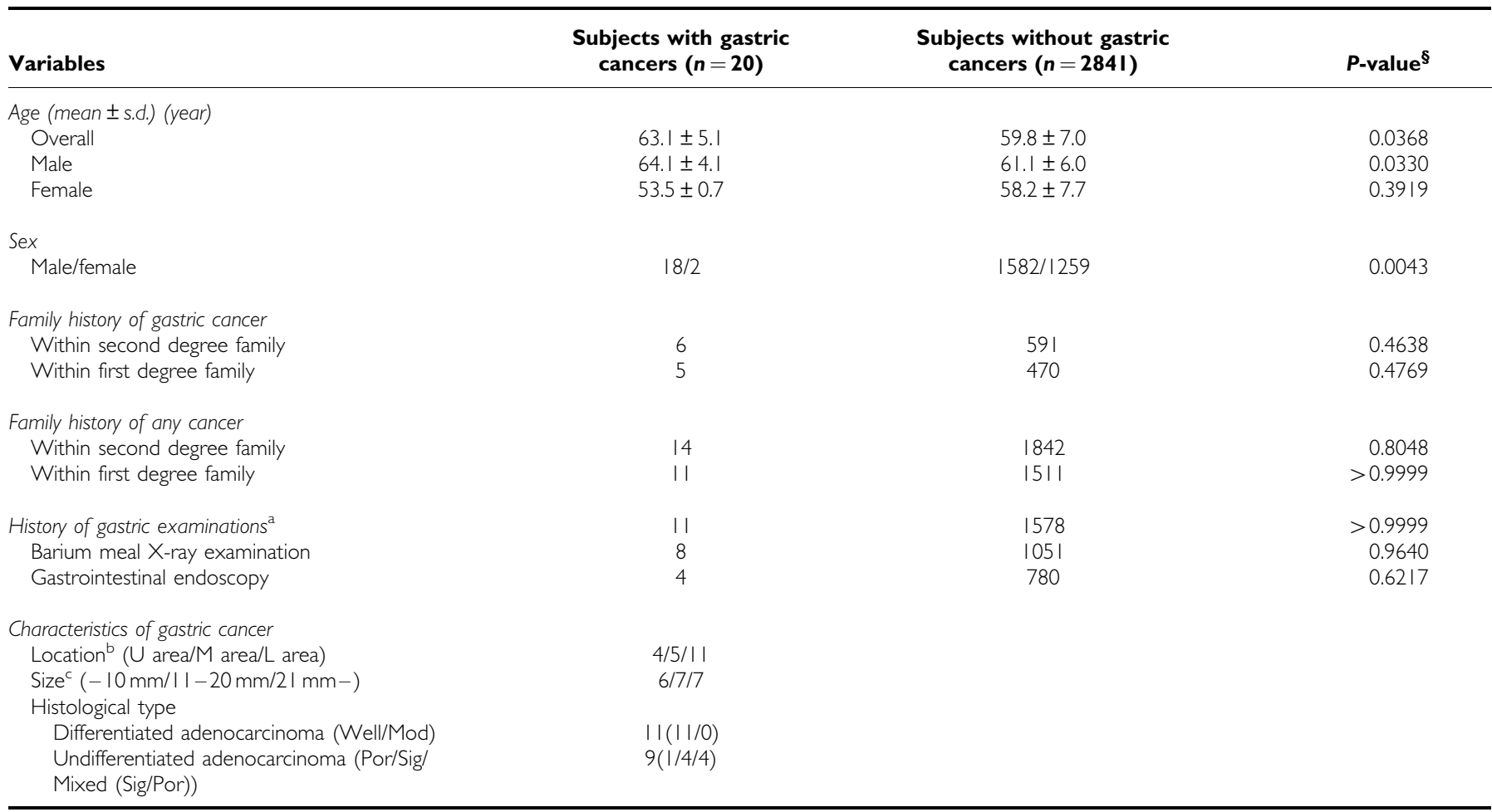

Mod = moderately differentiated adenocarcinoma; Por = poorly differentiated adenocarcinoma; Sig = signet ring cell carcinoma; Well = well-differentiated adenocarcinoma

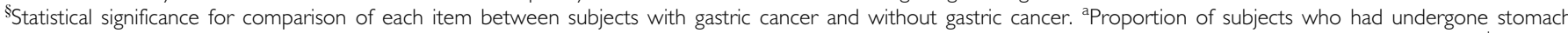
examination as a screening test or diagnostic test with X-ray examination and/or gastrointestinal endoscopy within I year before the screening endoscopy in this study. ${ }^{b}$ Location of a lesion is based on the 'Japanese Classification of Gastric Carcinoma' (The I3th Edition, 1999) by Japanese Gastric Cancer Association. 'Maximum diameter of cancer lesions.

Table 2 FDG-PET results according to depth of cancer invasion

\begin{tabular}{lccccc}
\hline & \multicolumn{4}{c}{ Depth of invasion $^{\mathbf{a}}$} \\
\cline { 3 - 6 } & & TI & T2 & T3 & T4 \\
\hline FDG-PET positive & $n=2$ & 1 & 0 & 0 & 1 \\
FDG-PET negative & $n=18$ & 17 & 1 & 0 & 0 \\
Total & $n=20$ & 18 & 1 & 0 & 1
\end{tabular}

FDG-PET denotes ${ }^{18}$ F-2-deoxy-2-fluoro-glucose positron emission tomography. TI: tumour invades lamina propria or submucosa. T2: tumour invades muscularis propria or subserosa. T3: tumour penetrates serosa (visceral peritoneum) without invasion of adjacent structures. T4: tumour invades adjacent structures. ${ }^{a}$ The depths of cancer invasion were based on the TNM classification.

There was no significant difference in the frequency of family history of gastric or any other cancer, or of previous examinations between subjects with gastric cancer and subjects without gastric cancer (Table 1).

Detailed clinical features of gastric cancers detected by endoscopy are shown in the bottom of Table 1. Histopathologically, about half of the cancers were well or moderately differentiated adenocarcinoma. Of the 20 gastric cancers, 18 were of T1 stage (Table 2), among which cancer invasion into the gastric wall was confined to the mucosa in 12 subjects, and to the submucosa in six subjects. Only two subjects among 20 cases with gastric cancer showed positive results with PET. The first patient had T4 cancer (Borrmann type 2, poorly differentiated adenocarcinoma), and the FDG-PET showed strong and focal accumulation in the area of the upper gastric body as 'positive pattern 1'. The second patient had T1 cancer (a superficial depressed type, signet
Table 3 Sensitivity and specificity of FDG-PET for gastric cancer

\begin{tabular}{lcc}
\hline & $\begin{array}{c}\text { Subjects with gastric } \\
\text { cancers }(\boldsymbol{n}=\mathbf{2 0})\end{array}$ & $\begin{array}{c}\text { Subjects without gastric } \\
\text { cancers }(\boldsymbol{n}=\mathbf{2 8 4} \mathbf{)})\end{array}$ \\
\hline $\begin{array}{l}\text { FDG-PET positive } \\
n=24\end{array}$ & 2 & 22 \\
$\begin{array}{l}\text { FDG-PET negative } \\
n=2837\end{array}$ & 18 & 2819 \\
\hline
\end{tabular}

$\mathrm{Cl}=$ confidence interval. Sensitivity $(95 \% \mathrm{Cl})=2 / 20=10 \%(1.2-31.7 \%)$. Specificity $(95 \% \mathrm{Cl})=28|9 / 284|=99.2 \%(98.8-99.5 \%)$. Positive predictive value $=2 / 24=8.3 \%$ $(1.0-27.0 \%)$. Negative predictive value $=2819 / 2837=99.4 \%(99.0-99.6 \%)$.

ring cell carcinoma), and the FDG-PET showed stronger accumulation in the area of the lower gastric body, which was clearly judged as 'positive pattern 2'.

The overall sensitivity, specificity, and positive predictive values were $10.0 \%$ (95\% CI: $1.2-31.7 \%$ ), $99.2 \%$ (95\% CI: $98.8-99.5 \%$ ), and $8.3 \%$ (95\% CI: $1.0-27.0 \%$ ), respectively, and the negative predictive value was $99.4 \%$ (95\% CI: 99.0-99.6\%) (Table 3). There were 22 subjects with positive FDG- PET accumulation in addition to two cases of gastric cancer. These 22 subjects had no other neoplastic lesions detected in the colon, nor in the other abdominal organs by colonoscopy and ultrasound sonography.

We compared the SUV between FDG-PET true positives (two subjects) and FDG-PET false positives (22 subjects). The mean \pm s.d. of the SUVs was $4.9 \pm 1.46$ in true positives and $4.5 \pm 0.96$ in false positives, and there was no significant difference between them. 


\section{DISCUSSION}

We have shown that the sensitivity of FDG-PET for gastric cancer is as low as $10 \%$ in this study. Although the sensitivity of FDG-PET for gastric cancer has been reported in some studies to range from 60 to 94\% (Yeung et al, 1998; De Potter et al, 2002; Stahl et al, 2003; Yoshioka et al, 2003; Mochiki et al, 2004; Chen et al, 2005; Yun et al, 2005), the subjects used in those reports were primarily clinically diagnosed, preoperative, advanced cancer, or recurrent cancer cases, and thus the sensitivity values calculated in those studies may not represent screening sensitivity. Screening sensitivity can only be measured in an asymptomatic population, preferably by performing diagnostic examination such as endoscopy on all subjects in order to identify cancer subjects in the population. There have been no other studies that have evaluated the sensitivity of FDG-PET for gastric cancer in an asymptomatic population based on the findings of endoscopy as the gold standard.

There are a few issues to be addressed, which might have influenced the sensitivity calculated in this study. Firstly, our case series of screen-detected cancers consists largely of cancers in the early stages, and the proportion of more advanced cancers was very small ( 2 of 20 ) (Table 2 ). Our previous report showed a little higher detection rate of gastric cancer in men than expected, which suggested possible overdiagnosis among screen-detected cancers (Hamashima et al, 2006). The high proportion of early cancers, including those of overdiagnosis among screen-detected cancers, could be a reason for our low sensitivity. There is one study from Japan in which the sensitivity of FDG-PET for early gastric cancer could be calculated, although the subjects used were clinically diagnosed cancers. Mochiki et al (2004) reported that the sensitivity was $40 \%$ in gastric cancers of $\mathrm{T} 1$ stage subsequently treated surgically. Although detailed information on the depth of cancer invasion was not available in that paper, the case series in their report was estimated to be of a more invasive nature than those in the present study in terms of depth of invasion. Because the indication for surgical resection of gastric cancer in terms of depth of cancer invasion is submucosal or deeper invasion in Japan, the subjects with T1 stage cancers would have had submucosal invasion in their study. In the present study, 12 out of $18 \mathrm{~T} 1$ cancers were intramucosal cancer, which did not necessarily require surgery. This difference might explain the difference in sensitivity for early cancer detection between the two studies. However, even when intramucosal cancers were excluded from the calculation, the sensitivity was only $12.5 \%$ (one positive out of eight). Secondly, in our study, we performed chromoendoscopy on all screenees, which might have enhanced the ability to detect small cancer lesions. Thirty percent of cancer lesions were $10 \mathrm{~mm}$ or less in diameter (Table 1). In any case, the calculated sensitivity in this study might be underestimated due to potential overdiagnosis relevant to screen-detected cancer as mentioned above.

The FDG-PET procedure employed in this study is based on the standard method used in clinical practice, except for the criteria for assessment of cancer. PET findings were assessed according to the criteria, which we defined, due to lack of established criteria. The main difficulty in FDG-PET diagnosis of stomach cancer is physiological uptake in the stomach (Cook et al, 1996; Gordon et al, 1997; Shreve et al, 1999; Koga et al, 2003), but there was no cancer subject in whom we had difficulty in differentiating physiological uptake from cancer lesions. Nevertheless, it is possible that there were tiny cancers overlooked due to significant FDG background uptake. As physiological uptake is more significant in the oral end of the stomach than in the anal end, screen-detected cancers with FDG-PET might be biased towards cancers in the anal end of the stomach.

In this study, there were 22 subjects with false-positive PET. There remains the possibility that upper gastrointestinal endoscopy had overlooked tiny lesions rather than that they were false positives. However, endoscopic images recorded in as many as approximately 50 shots were reviewed just after endoscopy to check for overlooked lesions. Therefore, it is unlikely that overlooked lesions were a main reason for such a low sensitivity.

It might be necessary to compare FDG-PET findings with those of existing examinations, such as barium meal and gastrointestinal endoscopy in terms of efficacy, cost, convenience, and radiation dose. Efficacy has been evaluated only for barium meal examinations in Japan by case-control studies (Oshima et al, 1986; Fukao et al, 1995; Mizoue et al, 2003). ${ }^{18} \mathrm{~F}$-2-deoxy-2-fluoro-glucose Positron Emission Tomography is more expensive than the other two procedures (85000 Japanese yen or 772 US\$ for FDG-PET, 12680 yen or 115 US\$ for endoscopy in our screening program, and about 82 US\$ for barium meal examination). There is much less inconvenience for screenees with FDG-PET than is seen after endoscopy or barium meal examination, which are often accompanied by discomfort during examination, side effects of antispasmodic agents, or constipation after examination. With regard to radiation dose, the average dose at our facility during the current study was $3.2 \mathrm{mSv}$ for FDG-PET and $4.4 \mathrm{mSv}$ for CT, which are similar to prior reports of barium meal examination that ranged from 3.0 to $9.3 \mathrm{mSv}$ (Broadhead et al, 1995; Geleijns et al, 1998), although the radiation dose of screening fluorography in Japan would be lower than barium meal examination as a diagnostic test (Kato et al, 1999).

This study did not evaluate the efficacy of FDG-PET screening for gastric cancer. Moreover, in this study, the sensitivity for more advanced cancers, which would be less likely to be affected by overdiagnosis, could not be measured due to an insufficient number of such cancers among screen-detected cancers. The sensitivity calculated here might thus be an underestimate of that for all gastric cancers. However, in conclusion, it was clearly demonstrated in this study that FDG-PET is poorly sensitive for the detection of gastric cancer in the early stages.

\section{ACKNOWLEDGEMENTS}

This study was supported in part by the Grants for Scientific Research Expenses for Health, Labour and Welfere Programs (H16-017, H16-020, and H18-003). We thank Mr D Kano for his help in synthesising FDG, Mr T Murano, Mr M Suzuki, for their technical assistance on performing PET, Mr H Sano for his contribution of statistical analysis, and $\mathrm{Mr} \mathrm{M}$ Kurashige for his technical support for endoscopy.

\section{REFERENCES}

Bombardieri E, Aktolun C, Baum RP, Bishof-Delaloye A, Buscombe J, Chatal JF, Maffioli L, Moncayo R, Mortelmans L, Reske SN (2003) FDGPET: procedure guidelines for tumour imaging. Eur J Nucl Med Mol Imaging 30: 115-124

Broadhead DA, Chapple CL, Faulkner K (1995) The impact of digital imaging on patient doses during barium studies. Br J Radiol 68: $992-996$
Chen J, Cheong JH, Yun MJ, Kim J, Lim JS, Hyung WJ, Noh SH (2005) Improvement in preoperative staging of gastric adenocarcinoma with positron emission tomography. Cancer 103: 2383-2390

Cook GJ, Fogelman I, Maisey MN (1996) Normal physiological and benign pathological variants of 18-fluoro-2-deoxyglucose positron-emission tomography scanning: potential for error in interpretation. Semin Nucl Med 26: $308-314$ 
De Potter T, Flamen P, Van Cutsem E, Penninckx F, Filez L, Bormans G, Maes A, Mortelmans L (2002) Whole-body PET with FDG for the diagnosis of recurrent gastric cancer. Eur J Nucl Med Mol Imaging 29: $525-529$

Fukao A, Tsubono Y, Tsuji I, Hisamichi S, Sugahara N, Takano A (1995) The evaluation of screening for gastric cancer in Miyagi Prefecture, Japan: a population-based case-control study. Int J Cancer 60: 45-48

Geleijns J, Broerse JJ, Chandie Shaw MP, Schultz FW, Teeuwisse W, Van Unnik JG, Zoetelief J (1998) A comparison of patient dose for examinations of the upper gastrointestinal tract at 11 conventional and digital X-ray units in The Netherlands. Br J Radiol 71: 745-753

Gordon BA, Flanagan FL, Dehdashti F (1997) Whole-body positron emission tomography: normal variations, pitfalls, and technical considerations. AJR Am J Roentgenol 169: 1675-1680

Hamashima C, Sobue T, Muramatsu Y, Saito H, Moriyama N, Kakizoe T (2006) Comparison of observed and expected numbers of detected cancers in the research center for cancer prevention and screening program. Jpn J Clin Oncol 36: $301-308$

Japanese Gastric Cancer Association (1999) Japanese Classification of Gastric Carcinoma, 13th edn, Tokyo: Kanehara

Kato H, Isobe T, Koshichi S, Okumura K, Kudo Y, Ishida Y, Kobayashi T, Takagi S, Anzai T, Hiroki S, Shiiba S, Nakamura H, Takemura T (1999) Evaluation of the difference in radiation dose among facilities in gastrointestinal X-ray examinations. Nippon Hoshasen Gijutsu Gakkai Zasshi 55: 655-664 Japanese

Kelloff GJ, Hoffman JM, Johnson B, Scher HI, Siegel BA, Cheng EY, Cheson BD, O'shaughnessy J, Guyton KZ, Mankoff DA, Shankar L, Larson SM, Sigman CC, Schilsky RL, Sullivan DC (2005) Progress and promise of FDG-PET imaging for cancer patient management and oncologic drug development. Clin Cancer Res 11: 2785-2808

Koga H, Sasaki M, Kuwabara Y, Hiraka K, Nakagawa M, Abe K, Kaneko K, Hayashi K, Honda H (2003) An analysis of the physiological FDG uptake pattern in the stomach. Ann Nucl Med 17: 733-738

Mizoue T, Yoshimura T, Tokui N, Hoshiyama Y, Yatsuya H, Sakata K, Kondo T, Kikuchi S, Toyoshima H, Hayakawa N, Tamakoshi A, Ohno Y, Fujino Y, Kaneko S (2003) Prospective study of screening for stomach cancer in Japan. Int J Cancer 106: $103-107$

Mochiki E, Kuwano H, Katoh H, Asao T, Oriuchi N, Endo K (2004) Evaluation of 18F-2-deoxy-2-fluoro-D-glucose positron emission tomography for gastric cancer. World J Surg 28: 247-253

Oku S, Nakagawa K, Momose T, Kumakura Y, Abe A, Watanabe T, Ohtomo $\mathrm{K}$ (2002) FDG-PET after radiotherapy is a good prognostic indicator of rectal cancer. Ann Nucl Med 16: $409-416$
Oshida M, Uno K, Suzuki M, Nagashima T, Hashimoto H, Yagata H, Shishikura T, Imazeki K, Nakajima N (1998) Predicting the prognoses of breast carcinoma patients with positron emission tomography using 2 deoxy-2-fluoro[18F]-D-glucose. Cancer 82: 2227-2234

Oshima A, Hirata N, Ubukata T, Umeda K, Fujimoto I (1986) Evaluation of a mass screening program for stomach cancer with a case-control study design. Int J Cancer 38: 829-833

Rigo P, Paulus P, Kaschten BJ, Hustinx R, Bury T, Jerusalem G, Benoit T, Foidart-Willems J (1996) Oncological applications of positron emission tomography with fluorine-18 fluorodeoxyglucose. Eur J Nucl Med 23: $1641-1674$

Shreve PD, Anzai Y, Wahl RL (1999) Pitfalls in oncologic diagnosis with FDG PET imaging: physiologic and benign variants. Radiographics 19: $61-77$; quiz 150-1

Sobin LH, Wittekind Ch (1997) TNM Classification of Malignant Tumors, 5 th edn Geneva: Wiley Liss

Stahl A, Ott K, Weber WA, Becker K, Link T, Siewert JR, Schwaiger M, Fink U (2003) FDG PET imaging of locally advanced gastric carcinomas: correlation with endoscopic and histopathological findings. Eur $\mathrm{J} \mathrm{Nucl}$ Med Mol Imaging 30: 288-295

Sugahara N, Hirasawa Y, Morimoto T, Sibuki S, Kogane T, Sato H, Fukao A, Yanbe T (1992) An investigative report about health state of old age groups at first screening for gastric mass survey. J Gastroenterol Mass Surv 95: 184-186 Japanese

Tamura K, Hayami H, Suzuki S, Saito F (1985) Use of Sodium picosulfate for constipation after barium meal examination in the screening program for gastric cancer. J Gastroenterol Mass Surv 69: 92-101 Japanese

World Health Organization Statistics, M.D., Vital Statistics and Information Department Ministry of Health, Labour and Welfare (2006) International Comparison of Cancer Mortality by Sex and Site

Yasuda S, Ide M (2005) PET and cancer screening. Ann Nucl Med 19: $167-177$

Yeung HW, Macapinlac H, Karpeh M, Finn RD, Larson SM (1998) Accuracy of FDG-PET in gastric cancer. Preliminary experience. Clin Positron Imaging 1: $213-221$

Yoshioka T, Yamaguchi K, Kubota K, Saginoya T, Yamazaki T, Ido T, Yamaura G, Takahashi H, Fukuda H, Kanamaru R (2003) Evaluation of $18 \mathrm{~F}-\mathrm{FDG}$ PET in patients with a, metastatic, or recurrent gastric cancer. J Nucl Med 44: 690-699

Yun M, Lim JS, Noh SH, Hyung WJ, Cheong JH, Bong JK, Cho A, Lee JD (2005) Lymph node staging of gastric cancer using (18)F-FDG PET: a comparison study with CT. J Nucl Med 46: 1582-1588 